\title{
Prevalencia del Estrés Asociado a la Doble Presencia y Factores Psicosociales en Trabajadores Estudiantes Chilenos
}

\author{
PREVALENCE OF STRESS ASSOCIATED TO THE DOUBLE PRESENCE AND PSYCHOSOCIAL FACTORS IN \\ WORKERS CHILEAN STUDENTS
}

Hernán Alejandro Fernández Espejo1', Guido Clemente Solari Montenegro

1. Universidad de Antofagasta. Chile.

\section{RESUMEN}

El estrés académico es una problemática frecuente en estudiantes universitarios, más aun cuando comparten responsabilidades académicas, trabajo y obligaciones en el hogar. La doble o triple presencia, es una condición frecuente en las ciudades del norte chileno caracterizadas por una alta empleabilidad en la gran minería del cobre, que obliga a hombres y mujeres trabajadores a estudiar en horarios vespertinos y/o nocturnos. El presente artículo tiene el propósito de determinar en aquella población que estudia y trabaja la relación entre la doble presencia, factores psicosociales laborales y la prevalencia de estrés. Mediante un diseño descriptivo se exploró 85 sujetos utilizando el cuestionario Suceso Istas-21, el Inventario SISCO de estrés académico y una encuesta sociodemográfica estandarizada. Los resultados refieren que la mayoría de las dimensiones psicosociales estudiadas presentan relaciones entre sí, que el estrés académico tiene una fuerte asociación con la doble presencia, las compensaciones, las exigencias psicológicas y el apoyo social y que las mujeres se manifiestan un nivel más alto de riesgo psicosocial y de estrés académico que los hombres por la dimensión doble presencia. Los síntomas más prevalentes en los sujetos estudiados fueron la alteraciones del sueño, apetito, inquietud y forma de afrontamiento. Tanto en hombres como en mujeres, los autores reconocen que el riesgo psicosocial más relevante lo constituye la dimensión doble presencia, particularmente en el género femenino y razonablemente suponen que la información generada constituye una herramienta a considerar en la gestión de salud de las organizaciones laborales para resguardo de la integridad de sus trabajadores.

(Fernández H, Solari G, 2017. Prevalencia del Estrés Asociado a la Doble Presencia y Factores Psicosociales en Trabajadores Estudiantes Chilenos. Cienc Trab. Sep-Dic; 19 [60]: 194-199).

Palabras claves: ESTRÉS, SALUD LABORAL.

\section{ABSTRACT}

Academic stress is a problem prevalent in university students, even more so when they share academic responsibilities, work and household obligations. The double or triple presence, is a very frequent condition in the cities of northern Chile, characterized by a high employability in the large-scale mining of copper, of which obliges men and women workers to study at evening or night hours. The purpose of this article was to determine the correlation between the double presence, psychosocial work factors and the prevalence of stress in that population. Using a descriptive design was explored 85 subjects using the questionnaire Istas-21 event, inventory SISCO of academic stress and a survey standardized demographic. The results indicate that most of the studied psychosocial dimensions present relations among themselves, academic stress has a strong association with the double presence, compensation, psychological demands and social support and that women show a higher level of psychosocial risk and stress academic than men by the dimension double presence. The symptoms most prevalent in the subjects studied were the alterations in sleep, appetite, restlessness, and way of coping. Both men and women, the authors acknowledge that the most relevant psychosocial risk double dimension constitutes presence, particularly in the female gender and they reasonably assume that the information generated is a tool to consider in the management of health of labor organizations to protect the integrity of their workers.

Key words: STRESS, OCCUPATIONAL HEALTH.

\section{INTRODUCCIÓN}

En un mundo altamente competitivo los trabajadores están cada vez más conscientes de la importancia que tiene el perfeccionamiento continuo. En el pasado el trabajador con un empleo estable, no consideraba el imperativo de volver a estudiar, sin embargo en los últimos años esta tendencia ha ido cambiando. Según un estudio realizado el año 2012 por la red latinoamericana de universidades (Universia) y en el que participaron más de 13000 universitarios de Argentina, Brasil, Colombia, Chile, 
España, México, Perú, Portugal, Puerto Rico y Uruguay, se concluyó que el 67\% de los estudiantes universitarios compartían las clases con su primer empleo, y que siete de cada diez los estudiantes trabajaban y estudiaban a la vez. ${ }^{1}$

El empleo y el estudio simultáneos, constituyen responsabilidades que con frecuencia representan exigencias cuantitativas y cualitativas que se podrían vincular con el estrés de las personas que las deben experimentar. Según la Organización Mundial de la Salud $(\mathrm{OMS})^{2}$, el estrés constituye un conjunto de reacciones fisiológicas que preparan al organismo para la acción, por otra parte, Trucco $^{3}$ lo definió como un conjunto de procesos y respuestas neuroendocrinas, inmunológicas, emocionales y conductuales ante situaciones que significan una demanda de adaptación mayor y a su vez una amenaza. Se trata de una reacción de activación fisiológica, emocional, cognitiva y conductual ante estímulos y eventos generados a nivel educativo, y para cuyo análisis Barraza ${ }^{4}$ propone tres ámbitos, uno centrado en los estresores, otro en las manifestaciones clínicas y finalmente en las estrategias de afrontamiento, es por eso que resulta razonable que el estrés en los estudiantes se debería analizar integralmente, desde la exposición a diversos estímulos como los horarios de clases, los excesos de tareas o las características del profesor, hasta la frecuencia de algunos síntomas, sin embargo también pueden existir factores psicosociales laborales que influyan en el estudiante trabajador, es decir condiciones en el contexto de su trabajo que lo afecten, motivo central de ésta investigación y así profundizar en su conocimiento.

La doble presencia, o situación social de desempeño en dos frentes simultáneamente, es un factor de riesgo interesante a la hora de valorar cómo afecta al estudiante, y cuáles son las circunstancias más frecuentes con las que se asocia. La valoración de la doble presencia se ha estudiado en la mujer que trabaja y se han reportados resultados observados en estudios centrados en el conflicto trabajo-familia en mujeres profesionales ${ }^{5}$ los que concluyeron que la sobrecarga de rol laboral y familiar, generaba conflictos en este último y cuyas causas eran ciertas condiciones laborales (carga de trabajo y formas de contratación) y la doble jornada de trabajo. En Chile el tema el tema relevante considerando los datos publicados por el Consejo Nacional de Educación (CNED), que estimó para el año 2016 un total de 1.161.222 alumnos matriculados en la educación superior, valor que duplica las cifras de hace una década atrás. ${ }^{6}$ También en Chile, pero el año 2013, el Instituto Nacional de la Juventud (INJ) publicó la Encuesta Nacional de la Juventud, señalando que uno de cada tres estudiantes de educación superior en el país estudiaba y trabajaba al mismo tiempo, especificando que en la región de Antofagasta el 42\% de los sujetos compatibilizaba los roles de trabajo y estudio simultáneos. ${ }^{7}$

Por otra parte, la valoración de las características sociodemográficas y el estrés académico ha sido poco explorada en estudios simultáneos mientras la dimensión laboral recobra importancia considerando que en Chile se ha relevado el rol de los factores psicosociales bajo el argumento que las exigencias psicológicas, posibilidades de desarrollo, control sobre el tiempo de trabajo, apoyo social en la empresa, calidad de liderazgo, conflictos de rol o compensaciones y doble presencia, influyen directamente en la salud del trabajador.

La $\mathrm{OMS}^{8}$ en el 2010 reportó a Chile como uno de los países con mayor carga de morbilidad por enfermedades psiquiátricas $(23,2 \%)$ en el mundo, y evidenció un problema que constituye un desafío constante para la Salud Pública del país en razón de generar políticas de intervención a nivel académico y laboral en todos sus niveles. Así lo menciona el convenio de la Organización Internacional del Trabajo (OIT) $\mathrm{N}^{\circ} 187$ al proponer el año 2013, que cada país debe identificar nuevos y emergentes peligros en el lugar de trabajo particularmente los de naturaleza psicosocial para favorecer los entornos seguros y saludables. ${ }^{9}$

Moreno ${ }^{10}$, refiere en su artículo titulado "Los riesgos laborales psicosociales, marco conceptual y contexto socio-económico", que dichos riesgos psicosociales pueden ser consecuencia de la organización del trabajo y que tienen una alta probabilidad de afectar negativamente la salud del trabajador, entendiendo que no derivan necesariamente en daño, pero que dependen de la susceptibilidad personal del individuo, experiencia, historia y contexto en que se desenvuelve la persona y que los factores de riesgo psicosocial se asocian a interacciones entre el trabajo, el medio ambiente, la satisfacción laboral y condiciones de su organización, además de las capacidades, necesidades, cultura y situación personal del trabajo fuera del ambiente laboral. La OIT ${ }^{11}$ coincide con algunos estudios especializados de salud y seguridad en el trabajo a la hora de identificar cinco grandes grupos de riesgos psicosociales: El exceso de exigencias psicológicas del trabajo, la falta de control, la influencia y desarrollo, la falta de apoyo social y de calidad de liderazgo, las escasas compensaciones del trabajo y la doble jornada o doble presencia. Lo anterior coincide en lo afirmado por la $\mathrm{OMS}^{8}$ en el documento "Modelo de Organizaciones Saludables", que asocia las causales de los riesgos en la organización del trabajo con los estilos de liderazgo, la aplicación y protección inconsistente de los derechos básicos de los trabajadores y las dificultades de afrontar el rol domestico-laboral, sobre todo durante los sistemas de turnos rotatorios.

Actualmente el escenario laboral chileno está marcado por la polifuncionalidad de los trabajadores, la demanda organizacional de cumplir metas productivas y la inestabilidad laboral. La inestabilidad en el empleo y el nivel de remuneraciones per cápita que aún se diferencia del promedio de países de la Organización para la Cooperación y el Desarrollo Económicos (OCDE) ${ }^{12}$, hacen que Chile sea un pais altamente desigual en cuestión de ingreso, riqueza y educación. De hecho, en la ENETS, Encuesta nacional de empleo, trabajo, salud y calidad de vida de los trabajadores y trabajadoras en Chile del Ministerio de Salud de Chile 2009$2010^{13}$ se reconoce que un $20 \%$ de trabajadores dependientes percibe su situación laboral como inestable y que la ausencia de contratos de trabajo llega a un 10\% en el caso de las mujeres y un $5 \%$ en los varones, la misma encuesta reveló que los principales sintomas que se asociaban al trabajo eran la sensación continúa de cansancio, dolor de cabeza, problemas para dormir y sentirse tenso o irritable, no obstante lo anterior los trabajadores (as) referían que casi siempre disfrutaban de su trabajo.

Por otra parte, la Superintendencia de Seguridad Social (SUSESO), mediante la Unidad de Riesgo Psicosocial Laboral dispuso en noviembre del año 2013, la versión revisada de una herramienta, SUSESO Istas 2114 para la gestión de riesgos psicosociales y de modo complementario el MINSAL, mediante el Instituto de Salud Pública, dispuso el año 2012 un Instrumento de evaluación de medidas para la prevención de riesgos psicosociales en el trabajo, instrumento de origen canadiense que valora 169 preguntas distribuidas en 16 dimensiones y permite evaluar las medidas de prevención de riesgos psicosociales en el trabajo, es decir las gestiones que ha realizado la empresa en dicha materia. ${ }^{15}$ 


\section{OBJETIVO}

Con todo, este artículo tiene el propósito de Identificar la relación entre la frecuencia de estrés académico, la doble presencia y los factores psicosociales laborales en un grupo de trabajadores que estudian en el Plan especial de pregrado para trabajadores de la Universidad

\section{JUSTIFICACIÓN DEL TRABAJO}

Identificar de los factores asociados al estrés académico en un grupo de trabajadores que estudian, permite ordenar la información respecto de las variables comprendidas en el estrés y aquellas contenidas en la doble presencia y factores psicosociales que coexisten en los hombres y mujeres trabajadoras. De relevancia es la magnitud de los trabajadores que estudian en la región del norte de Chile, no así los estudios destinados a esclarecer el contexto y potenciales agentes de riesgos que podrían afectar a hombres y mujeres en su estudio, en su trabajo y/o en su vida familiar. Los autores esperan que la información derivada del presente estudio sirva de insumo para generar nuevos y más extensos estudios de investigación en el tema, para reflexionar sobre medidas de gestión de riesgos psicosociales que mediante la promoción, prevención y control resulten en una mejor calidad de vida de las personas y que se releve el tema de propender a generar ambientes laborales saludables como condicionante para humanizar el trabajo, el estudio y la familia.

\section{METODOLOGIA}

Mediante un diseño descriptivo de alcance correlacional se exploró a 85 trabajadores que conformaron una muestra seleccionada por conveniencia de un total de 109 sujetos que estudiantes del Plan especial de pregrado para trabajadores de la Universidad Nacional Arturo Prat con sede en la ciudad de Calama, Chile. Los criterios de inclusión utilizados para seleccionar a los sujetos fueron: Que trabajaran y estudiaran simultáneamente (doble presencia) y que tuvieran entre 18 y hasta 55 años de edad.

Los autores consideraron el estrés académico como variable
Tabla 1.

Distribución porcentual de trabajadores con y sin estrés académico.

$\begin{array}{llllll} & & \text { Frecuencia } & \text { Porcentaje } & \begin{array}{l}\text { Porcentaje } \\ \text { válido }\end{array} & \begin{array}{l}\text { Porcentaje } \\ \text { acumulado }\end{array} \\ \text { ESTRES } & \text { NO } & 4 & 4,7 & 4,7 & 4,7 \\ & \text { SI } & 81 & 95,3 & 95,3 & 100,0 \\ & \text { Total } & 85 & 100,0 & 100,0 & \end{array}$

dependiente y lo valoraron mediante el Inventario SISCO de estrés académico, (Anexo 1). Otras 12 variables fueron consideradas variables independientes y se estudiaron como probables causas, a saber: Los factores psicosociales (valorados mediante el cuestionario SUSESO - ISTAS 21, Versión breve, (Anexo 2), la doble presencia (valorada mediante el cuestionario SUSESO ISTAS 21). $\mathrm{Y}$ finalmente el sexo, edad, trabajo activo, rubro o actividad económica, horas semanales de trabajo, tipo de turno, tipo de contrato, rol o actividades domésticas, horas de trabajo diario y tipo de horario/turno, fueron explorados mediante una Encuesta sociodemográfica estructurada (Anexo 3)

En el estudio se consideró todas las recomendaciones éticas contenidas en "Protocolo para estudios en seres humanos" disponible en la página web de la universidad de Antofagasta, Comité de Ética e investigación científica de la Universidad de Antofagasta (CEIC UA). Cada sujeto se incorporó al estudio una vez que otorgara su consentimiento informado (Anexo 4) y voluntariedad de participación o abandono.

Los datos recopilados por uno de los investigadores fueron posteriormente registrados a una planilla del software EXCEL (versión 2010) y posteriormente fueron analizados a ciegas por un tercer investigador con apoyo del programa IBM SPSS (Versión 24) y presentando los resultados más relevante mediante tablas para facilitar el análisis de los resultados .

\section{RESULTADOS}

Según lo expuesto en la tabla 2 puede afirmar que existe relación entre el estrés académico y la doble presencia. El factor psicosocial que presentó mayor asociación positiva y significativa con el estrés académico fue la doble presencia $(\mathrm{Rho}=0,340 \mathrm{p}<0.01$ ), seguido por compensaciones, exigencias psicológicas ( $\mathrm{Rho}=0,331$ $\mathrm{p}<0,01 ; \mathrm{Rho}=0,293 \mathrm{p}<0.01)$ y finalmente apoyo social $(\mathrm{Rho}=0,225$

Tabla 2.

Matriz de correlaciones entre factores psicosociales laborales y estrés académico.

\begin{tabular}{|c|c|c|c|c|c|c|c|c|}
\hline & & & \multicolumn{6}{|c|}{ Matriz de Correlaciones } \\
\hline & & & E.A & E.Ps & T.A & A.S & COM & D.P \\
\hline & \multirow[t]{2}{*}{ E.A } & \multirow{2}{*}{$\begin{array}{l}\text { Coeficiente de correlación } \\
\text { Sig. (bilateral) }\end{array}$} & 1,000 & \multirow{2}{*}{$\begin{array}{c}0,293^{* *} \\
0,008\end{array}$} & \multirow{2}{*}{$\begin{array}{c}-0,020 \\
0,858\end{array}$} & \multirow{2}{*}{$\begin{array}{l}0,225^{*} \\
0,044\end{array}$} & \multirow{2}{*}{$\begin{array}{c}0,331^{* *} \\
0,003\end{array}$} & \multirow{2}{*}{$\begin{array}{r}0,340^{* *} \\
0,002\end{array}$} \\
\hline & & & . & & & & & \\
\hline & \multirow[t]{2}{*}{ E.Ps } & \multirow{2}{*}{$\begin{array}{l}\text { Coeficiente de correlación } \\
\text { Sig. (bilateral) }\end{array}$} & \multirow{2}{*}{$\begin{array}{c}0,293^{* *} \\
0,008\end{array}$} & 1,000 & \multirow{2}{*}{$\begin{array}{c}-0,066 \\
0,561\end{array}$} & \multirow{2}{*}{$\begin{array}{c}0,424^{* *} \\
0,000\end{array}$} & \multirow{2}{*}{$\begin{array}{c}0,295^{* *} \\
0,008\end{array}$} & \multirow{2}{*}{$\begin{array}{l}0,190 \\
0,090\end{array}$} \\
\hline & & & & . & & & & \\
\hline \multirow{9}{*}{ Rho de Spearman } & \multirow[t]{2}{*}{ T.A } & \multirow{2}{*}{$\begin{array}{l}\text { Coeficiente de correlación } \\
\text { Sig. (bilateral) }\end{array}$} & \multirow{2}{*}{$\begin{array}{c}-0,020 \\
0,858\end{array}$} & \multirow{2}{*}{$\begin{array}{c}-0,066 \\
0,561\end{array}$} & 1,000 & \multirow{2}{*}{$\begin{array}{c}0,281^{*} \\
0,011\end{array}$} & \multirow{2}{*}{$\begin{array}{c}0,301^{* *} \\
0,007\end{array}$} & \multirow{2}{*}{$\begin{array}{c}-0,104 \\
0,355\end{array}$} \\
\hline & & & & & . & & & \\
\hline & \multirow[t]{2}{*}{ A.S } & \multirow{2}{*}{$\begin{array}{l}\text { Coeficiente de correlación } \\
\text { Sig. (bilateral) }\end{array}$} & $0,225^{*}$ & $0,424^{* *}$ & $0,281^{*}$ & 1,000 & \multirow{2}{*}{$\begin{array}{c}0,365^{* *} \\
0,001\end{array}$} & \multirow{2}{*}{$\begin{array}{l}0,139 \\
0,214\end{array}$} \\
\hline & & & 0,044 & 0,000 & 0,011 & . & & \\
\hline & \multirow[t]{2}{*}{ COM } & \multirow{2}{*}{$\begin{array}{l}\text { Coeficiente de correlación } \\
\text { Sig. (bilateral) }\end{array}$} & $0,331^{* *}$ & $0,295^{* *}$ & $0,301^{* *}$ & \multirow{2}{*}{$\begin{array}{c}0,365^{* *} \\
0,001\end{array}$} & 1,000 & \multirow{2}{*}{$\begin{array}{l}0,138 \\
0,223\end{array}$} \\
\hline & & & 0,003 & 0,008 & 0,007 & & . & \\
\hline & \multirow[t]{2}{*}{ D.P } & \multirow{2}{*}{$\begin{array}{l}\text { Coeficiente de correlación } \\
\text { Sig. (bilateral) }\end{array}$} & $0,340^{* *}$ & 0,190 & $-0,104$ & 0,139 & \multirow{2}{*}{$\begin{array}{l}0,138 \\
0,223\end{array}$} & \multirow{2}{*}{1,000} \\
\hline & & & 0,002 & 0,090 & 0,355 & 0,214 & & \\
\hline & & $\mathrm{N}$ & 81 & 81 & 81 & 81 & 80 & 81 \\
\hline
\end{tabular}

** La correlación es significativa en el nivel 0,01 (bilateral). * . La correlación es significativa en el nivel 0,05 (bilateral).

E.A=Estrés académico; E.Ps=Exigencias Psicológicas; T.A=Trabajo Activo; $\quad A . S=A p o y o$ Social; $C O M=$ Compensaciones; D.P=Doble presencia 
$\mathrm{p}<0,05)$. La asociación de la dimensión trabajo activo, fue negativa y no significativa (Rho $=-0.020 \mathrm{p}>0.05)$. En general, se observa que la mayoría de las dimensiones psicosociales presentan relaciones entre sí, destacándose la de apoyo social y exigencias psicosociales (Rho=0,424 $\mathrm{p}<0,01$ ).

Según lo expuesto en la tabla 2 puede afirmar que existe relación entre el estrés académico y la doble presencia. El factor psicosocial que presentó mayor asociación positiva y significativa con el estrés académico fue la doble presencia ( $R h o=0,340 \mathrm{p}<0.01$ ), seguido por compensaciones, exigencias psicológicas (Rho=0,331 $\mathrm{p}<0,01$; $\mathrm{Rho}=0,293 \mathrm{p}<0.01$ ) y finalmente apoyo social ( $\mathrm{Rho}=0,225 \mathrm{p}<0,05)$. La asociación de la dimensión trabajo activo, fue negativa y no significativa $(\mathrm{Rho}=-0.020 \mathrm{p}>0.05)$. En general, se observa que la mayoría de las dimensiones psicosociales presentan relaciones entre sí, destacándose la de apoyo social y exigencias psicosociales (Rho=0,424 $\mathrm{p}<0,01$ ).

De todos los sujetos con RIESGO PSICOSOCIAL ALTO $(n=58)$ en la dimensión doble presencia, un 82,8\% tiene estrés académico moderado, un 13,8\% leve y un 3,4\% presenta un estrés académico profundo; mientras que de los que presentan RIESGO PSICOSOCIAL MEDIO $(n=12)$ el 75\% tiene estrés moderado y el resto leve y

Tabla 3.

Distribución porcentual de niveles de estrés académico según dimensión doble presencia (SUSESO-Istas 21).

\begin{tabular}{|c|c|c|c|c|c|c|}
\hline & & & \multicolumn{3}{|c|}{ Niveles de Estrés Académico } & \multirow[b]{2}{*}{ TOTAL } \\
\hline & & & Leve & Moderado & Profundo & \\
\hline \multirow{9}{*}{$\begin{array}{l}\text { DOBLE } \\
\text { PRESENCIA }\end{array}$} & \multirow[t]{3}{*}{ ALTO } & \multirow{3}{*}{$\begin{array}{l}\text { Recuento } \\
\% \text { dentro de } \\
\text { doble presencia } \\
\% \text { del total }\end{array}$} & 848 & 2 & 58 & \\
\hline & & & $13,8 \%$ & $82,8 \%$ & $3,4 \%$ & $100,0 \%$ \\
\hline & & & $9,9 \%$ & $59,3 \%$ & $2,5 \%$ & $71,6 \%$ \\
\hline & \multirow{3}{*}{ BAJO } & \multirow{3}{*}{$\begin{array}{l}\text { Recuento } \\
\% \text { dentro de } \\
\text { doble presencia } \\
\% \text { del total }\end{array}$} & 55 & 1 & 11 & \\
\hline & & & $45,5 \%$ & $45,5 \%$ & $9,1 \%$ & $100,0 \%$ \\
\hline & & & $6,2 \%$ & $6,2 \%$ & $1,2 \%$ & $13,6 \%$ \\
\hline & \multirow[t]{3}{*}{ MEDIO } & \multirow{3}{*}{$\begin{array}{l}\text { Recuento } \\
\% \text { dentro de } \\
\text { doble presencia } \\
\% \text { del total }\end{array}$} & 39 & 0 & 12 & \\
\hline & & & $25,0 \%$ & $75,0 \%$ & $0,0 \%$ & $100,0 \%$ \\
\hline & & & $3,7 \%$ & $11,1 \%$ & $0,0 \%$ & $14,8 \%$ \\
\hline \multirow{3}{*}{\multicolumn{2}{|c|}{ TOTAL }} & \multirow{3}{*}{$\begin{array}{l}\text { Recuento } \\
\% \text { dentro de } \\
\text { doble presencia } \\
\% \text { del total }\end{array}$} & 1662 & 3 & 81 & \\
\hline & & & $19,8 \%$ & $76,5 \%$ & $3,7 \%$ & $100,0 \%$ \\
\hline & & & $19,8 \%$ & $76,5 \%$ & $3,7 \%$ & $100,0 \%$ \\
\hline
\end{tabular}

Tabla 4.

Distribución porcentual de niveles de riesgo de la dimensión doble presencia según sexo

\begin{tabular}{|c|c|c|c|c|c|}
\hline & & & HOMBRE & MUJER & TOTAL \\
\hline \multirow{12}{*}{$\begin{array}{l}\text { RIESGO } \\
\text { DE DOBLE } \\
\text { PRESENCIA }\end{array}$} & \multirow[t]{4}{*}{ ALTO } & Recuento & 19 & 38 & 57 \\
\hline & & $\begin{array}{l}\% \text { dentro de riesgo } \\
\text { doble presencia }\end{array}$ & $33,3 \%$ & $66.7 \%$ & $100,0 \%$ \\
\hline & & $\%$ dentro de sexo & $55,9 \%$ & $82,6 \%$ & $71,25 \%$ \\
\hline & & $\%$ del total & $23,75 \%$ & $47,5 \%$ & $71,25 \%$ \\
\hline & \multirow[t]{4}{*}{ MEDIO } & Recuento & 7 & 5 & 12 \\
\hline & & $\begin{array}{l}\% \text { dentro de riesgo } \\
\text { doble presencia }\end{array}$ & $58,3 \%$ & $41,7 \%$ & $100,0 \%$ \\
\hline & & $\%$ dentro de sexo & $20,6 \%$ & $10,9 \%$ & $15 \%$ \\
\hline & & $\%$ del total & $8,75 \%$ & $6,25 \%$ & $15 \%$ \\
\hline & \multirow[t]{4}{*}{ BAJO } & Recuento & 8 & 3 & 11 \\
\hline & & $\begin{array}{l}\% \text { dentro de riesgo } \\
\text { doble presencia }\end{array}$ & $72,7 \%$ & $27,3 \%$ & $100,0 \%$ \\
\hline & & $\%$ dentro de sexo & 23,5 & $6.52 \%$ & $13,75 \%$ \\
\hline & & $\%$ del total & $10 \%$ & $3,75 \%$ & $13,75 \%$ \\
\hline & TOTAL & Recuento & 34 & 46 & 80 \\
\hline
\end{tabular}

Tabla 5.

Relación doble presencia- sexo.

\begin{tabular}{|c|c|c|c|c|}
\hline & & ANE & & \\
\hline & Sexo & $\mathrm{N}$ & $\begin{array}{l}\text { Rango } \\
\text { Promedio }\end{array}$ & $\begin{array}{l}\text { Suma De } \\
\text { Rangos }\end{array}$ \\
\hline & HOMBRE & 34 & 34,35 & 1168,00 \\
\hline $\begin{array}{l}\text { DUBLE } \\
\text { PRESENCIA }\end{array}$ & MUJER & 46 & 45,04 & 2072,00 \\
\hline & TOTAL & 80 & & \\
\hline
\end{tabular}

$\begin{array}{lcc} & \text { ESTADISTICOS DE PRUEBA } & \\ & & \text { DP } \\ \text { U de Mann-Whitney } & 573,000 \\ \text { W de Wilcoxon } & 1168,000 \\ Z & -2,054 \\ \text { Sig. asint. (bilateral) } & 0,040\end{array}$

Tabla 6.

Distribución porcentual de niveles de estrés académico de los trabajadores.

\begin{tabular}{llcccc} 
& & Frecuencia & Porcentaje & $\begin{array}{c}\text { Porcentaje } \\
\text { Válido }\end{array}$ & $\begin{array}{c}\text { Porcentaje } \\
\text { Acumulado }\end{array}$ \\
\hline NIVEL DE & LEVE & 15 & 18,5 & 18,5 & 18,5 \\
ESTRÉS & MODERADO & 63 & 77,8 & 77,8 & 96,3 \\
& PROFUNDO & 3 & 3,7 & 3,7 & 100,0 \\
& TOTAL & 81 & 100,0 & 100,0 &
\end{tabular}

finalmente los con RIESG0 PSICOSOCIAL BAJO ( $n=11$ ), un 45,5\% tienen estrés académico leve y la misma cifra estrés académico moderado.

Del total de mujeres, el 82,6\% presenta riesgo alto (38 de 46), a diferencia de los varones donde el 55,9\% presenta esta condición (19 de 34).

En ambos géneros el nivel de riesgo predominante es el "ALTO".

De todos los sujetos que presentan RIESGO ALTO por la doble presencia el 66,7\% son mujeres y el 33,3 varones.

Ya que el valor obtenido (0.040) es menor a 0,05 se puede asumir que la doble presencia no depende del sexo del sujeto. La doble presencia y el género femenino se asocian solamente en el nivel de riesgo alto donde las mujeres representan el 82.6\% y los hombres solo el 55,9\%. Acorde al baremo utilizado en este estudio (Barraza 2007) de los trabajadores que tienen estrés, el 77,8\% presenta estrés moderado; el $18,5 \%$ estrés leve y solo un 3,7\% profundo.

La frecuencia de la respuesta de la pregunta número 19 nos indica que el 35,3\% de los trabajadores responde que SIEMPRE las tareas

Tabla 7.

Distribución porcentual de respuestas más comunes por preguntas de la dimensión doble presencia.

\begin{tabular}{lccc}
\hline PREGUNTA & $\begin{array}{c}\text { DIMENSIÓN DOBLE PRESENCIA } \\
\text { RESPUESTA } \\
\text { MÁS FRECUENTE }\end{array}$ & $\begin{array}{c}\text { INDIVIDUOS } \\
\text { (de un total de 85 } \\
\text { trabajadores estudiantes) }\end{array}$ & PORCENTAJE \\
\hline $\begin{array}{l}\text { 19. Si está ausente un } \\
\text { día de casa, las tareas } \\
\text { domésticas que realiza } \\
\text { ise quedan sin hacer? }\end{array}$ & SIEMPRE & 30 & $35,3 \%$ \\
$\begin{array}{l}\text { 20. Cuando está en el } \\
\text { trabajo, ¿piensa en las } \\
\text { exigencias domésticas } \\
\text { y familiares? }\end{array}$ & ALGUNAS VECES & 30 & $35,3 \%$ \\
\hline
\end{tabular}

Tabla 8.

Determinación de media de estresores académicos.

\begin{tabular}{|c|c|c|c|c|c|c|}
\hline Dimensión & $\mathrm{N}$ & $\begin{array}{c}\% \\
\text { Mínimo }\end{array}$ & $\begin{array}{c}\% \\
\text { Máximo }\end{array}$ & $\begin{array}{c}\% \\
\text { Media }\end{array}$ & Desviación & Frecuencia \\
\hline $\begin{array}{l}\text { ESTRESORES } \\
\text { ACADÉMICOS }\end{array}$ & 81 & 6,3 & 81,3 & 47 & 15,9 & $\begin{array}{c}\text { Algunas } \\
\text { Veces }\end{array}$ \\
\hline
\end{tabular}


domésticas se quedan sin hacer cuando está ausente, mientras que la frecuencia de la respuesta de la pregunta número 20 nos indica que el 35,3\% responde que ALGUNAS VECES se piensa en exigencias domésticas y familiares cuando está en el trabajo.

En la tabla precedente se observa la dimensión estresores, que compone la variable estrés académico, donde se muestra un porcentaje medio de $47 \%$ que al interpretarlo con el baremo específico indica que las demandas del ambiente académico (evaluaciones, tipo de trabajo, características del profesor etc.) se presentan en promedio "algunas veces" en los sujetos con estrés académico.

\section{DISCUSION}

Según los resultados obtenidos en este estudio, el 95,3\% de la población explorada presentó estrés académico concordando con algunas investigaciones chilenas como las de Flores ${ }^{16}$ y Jerez ${ }^{17}$, quienes reportaron cifras de 94,6\% y $98 \%$ de sujetos con estrés académico respectivamente; y de Barraza ${ }^{4}$ quien halló similares resultados en estudiantes universitarios mexicanos en los que el estrés académico fue percibido en la gran mayoría de los estudiantes (95\%).

En nuestra investigación de trabajadores estudiantes, el porcentaje promedio de estrés académico fue de 45,96\% expresando un nivel "moderado" de estrés, dato que coincide con los estudios de Oliván et al. ${ }^{18}$, Flores ${ }^{16}$ y Díaz y Jiménez. ${ }^{19}$

En el contexto del género, nosotros no hallamos una diferencia porcentual relevante entre hombres y mujeres (94,4\% y 95,8\% respectivamente) concordando con lo señalado por De Miguel y Hernández en 2006 (citado por Rodríguez ML) ${ }^{20}$ y por Barraza ${ }^{4}$, pero en contrario a lo reportado por Marty et $\mathrm{al}^{21} \mathrm{y}$ Muñoz ${ }^{22}$ quienes encontraron mayores niveles de estrés académico en mujeres, del mismo modo que Flores ${ }^{16} \mathrm{y}$ de Jerez $^{17}$ quienes describieron una diferencia de $8 \%$ entre géneros.

Dentro de los estresores más frecuentes, nosotros reportamos la "limitación de tiempo", "evaluación de profesores" y la "sobrecarga de tareas y trabajos académicos" concordando con otros investigadores de universidades chilenas como Oliván et $\mathrm{al}^{18}$, Flores ${ }^{16}$ y Jerez. ${ }^{17}$ Por otra parte, Heras ${ }^{23}$ reportó como el estresor más prevalente las "tareas y exámenes" y secundariamente a la "no comprensión de temas" y la "limitación del tiempo de trabajo". Otros investigadores como Cabanach y Souto-Gestal ${ }^{24}$ en su publicación del año 2016 señalaron a la "deficiencia del profesorado" como agente estresante académico más frecuente, en tanto que Román et $\mathrm{al}^{25}$ utilizando un instrumento para valorar el estrés autopercibido identificaron a la organización docente, la malla curricular y el proceso docente como los agentes más frecuentes.

En este estudio, los sintomas físicos reportados coinciden con aquellos más frecuentes descritos en la Encuesta nacional de empleo trabajo, salud y calidad de vida (ENETS) publicada por el Ministerio de Salud Pública de Chile el 2009-2010. ${ }^{13}$ A saber, según orden de frecuencia, los síntomas físicos más relevantes y referidos por los sujetos estudiados fueron la "somnolencia" y las "cefaleas", en los síntomas comportamentales fueron las "alteraciones de apetito" y en los psicológicos la "inquietud" y "desconcentración"; coincidiendo con el estudio de Oliván et $\mathrm{al}^{18}$ quienes en esta misma materia identificaron como síntomas protagónicos la "somnolencia" e "inquietud" y con el estudio de Flores ${ }^{18}$ quien reportó las mismas manifestaciones que en el presente estudio. No obstante, Heras ${ }^{23}$ halló que el "desgano de hacer labores" fue la reacción comportamental más frecuente en los sujetos de su estudio.

En otra materia, Bedoya-Lau ${ }^{26}$ identificó que la habilidad asertiva y la elaboración de un plan, eran las estrategias más frecuentes de afrontamiento del estrés en un grupo de estudiantes universitarios, concordando con el estudio de Palacio ${ }^{27}$ quien reportó que la estrategia de "planificación de tareas" como una de las formas más frecuentes de afrontamiento al estrés. En nuestro estudio las estrategias de afrontamiento más recurridas fueron la "búsqueda de información de la situación" y "elaboración del plan".

En relación a los niveles de riesgo psicosocial predominante hallados en nuestro estudio, podemos mencionar el nivel alto de riesgo para las dimensiones de exigencias psicológicas $(38,2 \%$ de los sujetos), apoyo social $(49,4 \%)$, compensaciones $(42,4 \%) \mathrm{y}$ doble presencia (71,8\%), y nivel bajo de riesgo psicosocial para la dimensión trabajo activo (42,4\%), cifras que concuerdan con la investigación de Ceballos et $\mathrm{al}^{28}$ quien describió predominancia del riesgo alto para las dimensiones de exigencias psicológicas y doble presencia, mientras que Bravo y Nazar ${ }^{29}$ identificaron un nivel alto de riesgo para las exigencias psicológicas, nivel bajo para la dimensión de trabajo activo y nivel medio para el resto de las dimensiones.

En materia de relación entre los factores psicosociales y estrés académico, el factor con mayor asociación positiva y significativa fue la doble presencia, seguido por compensaciones, exigencias psicológicas y apoyo social.

En el presente artículo la doble presencia constituyó el factor de riesgo más relevante, particularmente en los niveles altos de riesgo psicosocial $(82,6 \%$ de las mujeres y un 55,9\% de los hombres). Además, todos los sujetos con riesgo psicosocial alto y en la dimensión doble presencia, de ellos, un 82,8\% manifestó estrés académico moderado.

La relación entre la dedicación compartida a tareas domésticas y niveles de estrés académico fue altamente significativa.

La importancia de la doble presencia en el género femenino reportada en el presente artículo coincide con lo señalado en la encuesta chilena ENETS $^{13}$ y el reporte del INSHT de España ${ }^{30}$, en ambas publicaciones se mencionan las repercusiones que en el ámbito familiar y social afecta a las mujeres que trabajan.

\section{CONCLUSIONES Y RECOMENDACIONES}

El presente estudio describe y correlaciona variables para facilitar la comprensión de los factores académicos y extra académicos que mediante el estrés afectan a los trabajadores que estudian.

Los autores concluyen que el factor con mayor asociación positiva y significativa en materia de factores psicosociales y estrés académico, fue la doble presencia y que particularmente en las mujeres con niveles altos de calificación de riesgo psicosocial, la doble presencia constituyó el factor de riesgo más relevante. Resultó también altamente significativa la relación entre niveles de estrés académico y la dedicación compartida a tareas domésticas.

Los estresores más frecuentemente referidos por los probandos fueron la "limitación de tiempo", "evaluación de profesores" y la "sobrecarga de tareas y trabajos académicos" y las estrategias de afrontamiento que ellos frecuentemente utilizaron fueron la "búsqueda de información de la situación" y "elaboración del plan". 
No son frecuentes los estudios que exploran esta materia en grupos humanos, por ello la necesidad de investigar el tema constituye un imperativo para identificar el contexto en el que ocurre la problemática y para establecer medidas razonables de control con probabilidad de éxito. Los autores enfatizan la necesidad de investigar en otras muestras de hombres y mujeres, utilizando diseños descriptivos yo analíticos para generar información con impacto disciplinar y social, y esperan que este artículo constituya un aporte para generar ideas y acciones orientadas a mejorar la calidad de vida de los trabajadores.

\section{REFERENCIAS}

1. Universia-Trabajando.com. Encuesta Iberoamericana en estudiantes universitarios. Madrid: Universia; 2012.

2. Organización Mundial de Salud. Salud, población y desarrollo; documento de posición de la OMS [en línea]. Conferencia internacional sobre la población y el desarrollo. 5-7 sept 1994; El Cairo, Egipto. Ginebra: OMS; 1994 [citado nov 2017]. Disponible en: http://apps.who.int/iris/bitstream/10665/59635/1/WHO_ FHE_94.1_spa.pdf

3. Trucco, M. Estrés y trastornos mentales: aspectos neurobiológicos y psicosociales. Rev chil neuro-psiquiatr. 2002; 40(Supl.2):8-19.

4. Barraza, A. El campo de estudio del estrés: del Programa de Investigación Estímulo-Respuesta al Programa de Investigación Persona-Entorno. Rev Int Psicol [en línea] 2007 [citado sep 2017]; 8(2):1-30. Disponible en: http://www. revistapsicologia.org/index.php/revista/article/view/48/45

5. Álvarez A, Gómez I. Conflicto trabajo-familia, en mujeres profesionales que trabajan en la modalidad de empleo. Pensam Psicol. 2011; 9(16):89-106.

6. Consejo Nacional de Educación. CNED. Indices 2016: Tendencias matrícula en educación superior [en línea]. Santiago de Chile: CNED; 2016 [citado jun 2017]. Disponible en: https://www.cned.cl/sites/default/files/presentacion_indices_ junio_2016.pdf

7. Instituto Nacional de la Juventud. VII Encuesta Nacional de la Juventud. Santiago de Chile: Ministerio de Desarrollo Social; 2013.

8. Organización Mundial de Salud (OMS). Entornos laborales saludables: fundamentos y modelo de la OMS: contextualización, prácticas y literatura de apoyo [en linea]. Ginebra: OMS; 2010 [citado jun 2017]. Disponible en: http://www. who.int/occupational_health/evelyn_hwp_spanish.pdf

9. Organización Internacional del Trabajo (OIT). La organización del trabajo y los riesgos psicosociales: una mirada de género. Ginebra: OIT; 2013.

10. Moreno B. Los riesgos laborales psicosociales: marco conceptual y contexto socio- económico. ORP Journal. 2014; 1:4-18.

11. Organización Internacional del Trabajo (OIT). Los factores psicosociales en el trabajo: reconocimiento y control. Ginebra: OIT; 2014.

12. Organización para la Cooperación y el Desarrollo Económico (OCDE). Estudios económicos de la OCDE: Chile. Paris: OCDE; 2015.

13. Chile. Ministerio de Salud. Dirección del Trabajo e Instituto de Seguridad Laboral. Primera encuesta nacional de empleo, trabajo, salud y calidad de vida de los trabajadores y trabajadoras en Chile (ENETS 2009-2010). Santiago: MINSAL; 2011.

14. Chile. Superintendencia de Seguridad Social. Cuestionario de evaluación de riesgos psicosociales en el trabajo SUSESO/ISTAS21. Santiago de Chile: SUSESO; 2013.

15. Chile. Ministerio de Salud. Instrumento de evaluación de medidas para la prevención de riesgos psicosociales en el trabajo. Santiago de Chile: Instituto de Salud Pública; 2012

16. Flores $\mathrm{A}$, Jara J, Montecinos $\mathrm{C}$, Pavéz N. Nivel de estrés académico en estudiantes de la carrera de enfermería de la Universidad del Bio-Bío Chillán, durante el primer semestre del año académico 2012 [tesis]. Chillán: Universidad del Bío-Bío; 2012
17. Jerez $M$, Oyarzo $C$. Estrés académico en estudiantes del Departamento de Salud de la Universidad de Los Lagos Osorno. Rev chil neuro-psiquiatr. 2015; 53(3):149-157.

18. Oliván $B$, Boira $S$, López $Y$. Estrés y otros factores psicológicos asociados en estudiantes de fisioterapia. Fisioterapia. 2010; 33(1):19-24.

19. Diaz $Y$, Jiménez $A$. Estrés académico en adolescentes del nivel de enseñanza pre universitario [en línea]. Camagüey: Universidad de las Ciencias Médicas Carlos J. Finlay; 2013 [citado sep 2017]. Disponible en: http://www.codajic.org/ sites/www.codajic.org/files/32\%20-\%20ESTR\%C3\%89S\%20 ACAD\%C3\%89MICO\%20EN\%2OADOLESCENTES\%20\%20DEL \%20NIVEL \%20 DE\%20ENSE\%C3\%91ANZ_0.pdf

20. Rodriguez ML. Competencias académicas, reporte de estrés y salud en estudiantes universitarios. REPI. 2012; 15(2):553-574.

21. Marty $M$, Lavin $M$, Figueroa $M$, Larrain $D$, Cruz $C$. Prevalencia de estrés en estudiantes del área de la salud de la Universidad de los Andes y su relación con enfermedades infecciosas. Rev chil neuro-psiquiatr. 2005; 43(1):25-32.

22. Muñoz F. Estrés académico: Problemas y soluciones desde una perspectiva psicosocial. Huelva: Universidad de Huelva; 2003.

23. Heras $\mathrm{G}$. Factores causantes de estrés académico en estudiantes de primer año de medicina en Oaxaca. Jornadas de Educación Médica 2011, Facultad de Medicina y Cirugia-Universidad Regional del Sureste; oct 2011; Oaxaca, México. Oaxaca: URSE; 2011.

24. Cabanach R, Souto-Gestal A, Franco V. Escala de Estresores Académicos para la evaluación de los estresores académicos en estudiantes universitarios. Rev Iberoam Psicol Salud. 2016; 7(2):41-50.

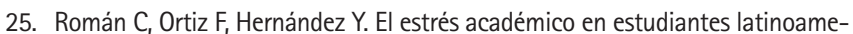
ricanos de la carrera de Medicina. Rev Iberoam Educ. 2008; 46(7):1-8.

26. Bedoya-Lau F, Matos L, Zelaya E. Niveles de estrés académico, manifestaciones psicosomáticas y estrategias de afrontamiento en alumnos de la facultad de medicina de una universidad privada de Lima en el año 2012. Rev neuropsiquiatr. 2014; 77(4):262-270.

27. Palacio J. Estrés académico y consumo de alcohol en los estudiantes de la Universidad de Antioquia, Seccional Occidente. Antioquia: Universidad de Antioquia; 2015.

28. Ceballos $P$, Rolo $G$, Hernández $E$, Díaz $D$, Paravic $T$, Burgos M. Factores psicosociales y carga mental de trabajo: Una realidad percibida por enfermeras (os) en unidades criticas. Rev Latino-Am Enfermagem. 2015; 23(2):315-322.

29. Bravo C, Nazar G. Riesgos psicosociales en el trabajo y salud en conductores de locomoción colectiva urbana en Chile. Salud Trab. 2015; 23(2):105-114.

30. Instituto Nacional de Seguridad e Higiene en el Trabajo (INSHT). Encuesta Nacional de Condiciones de Trabajo 2015, 6a EWCS España [en línea]. Madrid: INSHT; 2017 [citado sep 2017]. Disponible en: http://www.insht.es/InshtWeb/ Contenidos/Documentacion/FICHAS\%20DE\%20PUBLICACIONES/EN\%20 CATALOGO/GENERALIDAD/ENCT\%202015.pdf 